Super positi on of Si gnal Component s During I nspecti on of Printed Circuit Boards by an Eddy Cur rent Testing Probe wi th a Sol enoid Pi ck- up Coi I

\begin{tabular}{|l|l|}
\hline 著者 & $\begin{array}{l}\text { Kacpr zak D., Tani guchi T., Nakamur a N. Yamada } \\
\text { Sot oshi, I wahar a Masay oshi }\end{array}$ \\
\hline $\begin{array}{l}\text { j our nal or } \\
\text { publ i cat i on t i t l e }\end{array}$ & I EEE Tr ansact i ons on Nagget i cs \\
\hline vol une & 37 \\
\hline number & 4 \\
\hline page r ange & 2794 2796 \\
\hline year & $2001-07-01$ \\
\hline URL & ht t p: //hdl . handl e. net /2297/48309 \\
\hline
\end{tabular}




\title{
Superposition of Signal Components During Inspection of Printed Circuit Boards by an Eddy Current Testing Probe with a Solenoid Pick-up Coil
}

\author{
D. Kacprzak, T. Taniguchi, K. Nakamura, S. Yamada, Member, IEEE, and M. Iwahara, Member, IEEE
}

\begin{abstract}
This paper presents a theory of the superposition of two signal components formed during the inspection of printed circuit board (PCB) by an eddy-current testing probe composed of a meander-exciting coil and a solenoid pick-up coil. Proportion of these two components effects the amplitude and the phase of the output signal. Characteristic changes of the phase characteristic, obtained from calculations and observed during the real inspection of PCB, are explained.
\end{abstract}

Index Terms-Amplitude, components, meander coil, PCB testing, phase, signal, solenoid coil.

\section{INTRODUCTION}

D URING the inspection of Printed Circuit Board (PCB) by an eddy-current testing (ECT) probe, the information about possible defects can be obtained either from an amplitude characteristic or a phase characteristic. The ECT probe, used for the inspection, consists of a meander-exciting coil and a solenoid pick-up coil [1], [2]. The solenoid pick-up coil inspects a tangential component of the magnetic flux density, which appears only at the defect zones [3]. Analysis of the amplitude characteristic is mainly focused on a removal of an offset signal and a noise, measurement of signal's peaks and interpretation of a final signal [4], [5]. The analysis of the phase characteristic can also concern an analysis of a signal's shape, and it can be used in future for removal of soldering points from a final image [6]. In this paper, uncommon changes of the phase characteristic during the inspection are explained, A source of these changes is the superposition nature of the output signal. There are two components in the output signal, which self-proportion effects both: the amplitude and the phase characteristics of the output signal. A study of this phenomenon allows a new approach to the signal analysis, which is the analysis of a signal shape. Due to the specific nature of the phase characteristic, extraction of signals coming from elements such as soldering points is possible.

\section{StRuCture of THE ECT Probe}

Fig. 1 shows a highly sensitive ECT probe used for the inspection of PCB. A meander coil, used to inspect printed circuit boards, has a remarkable shape. Long conductors with an exciting current produce a magnetic field, which generates

Manuscript received October 13, 2000.

The authors are with the Faculty of Engineering, Kanazawa University, Kodatsuno 2-40-20, Kanazawa 920-8667, Japan (e-mail: kacprzak@magstar.ec.t.kanazawa-u.ac.jp).

Publisher Item Identifier S 0018-9464(01)07187-4.

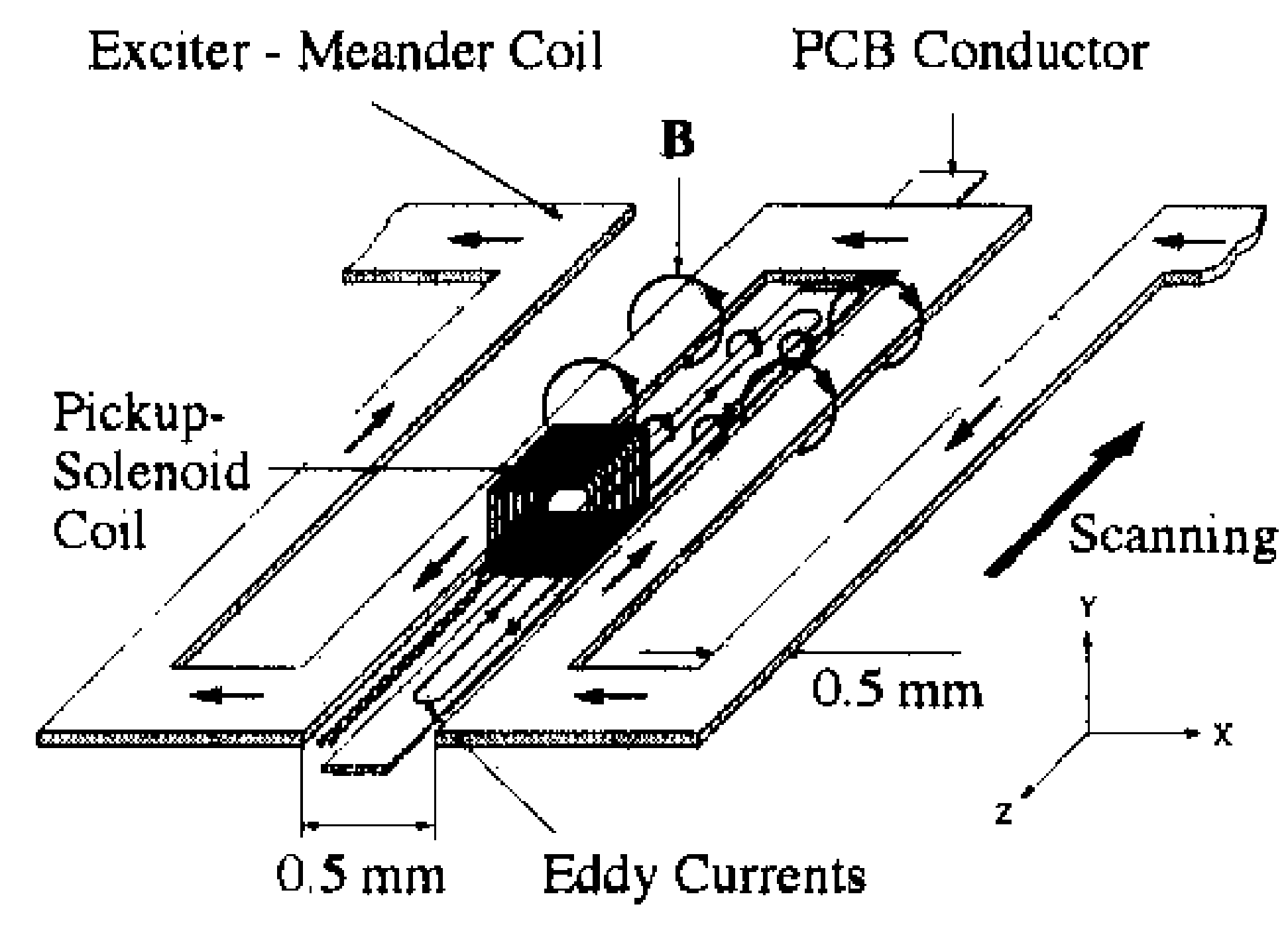

Fig. 1. ECT probe with solenoid pickup coil.

eddy currents in the PCB conductors. Any defects on the tested conductors appear as obstacles for the eddy currents and cause changes in their distributions. The key point of the ECT probe is its pick-up coil, which has a solenoid shape. This kind of pick-up coil allows an inspection of a tangential component of $B$. The inspection of the tangential component is more difficult than the inspection of the normal component, because the tangential component appears at a very small distance from the test conductor, and it's value is not as high as the value of the normal component. However, the tangential component appears only in specific cases-together with defects or at conductors boundaries. In the case of a defectless conductor, there is no tangential component generated. The output signal does not have to be extracted from others signals as in the case of the inspection of the normal component. This is a big advantage of the probe with a solenoid pick-up coil and the main reason for its high sensitivity. During scanning, the probe moves above the test PCB with constant lift-off. The exciting current frequency is $f=5 \mathrm{MHz}$. Resultant data, obtained from an lock-in amplifier are stored in a PC computer. Inspection can be performed along or across conductors. However, due to the specific shape of the meander coil and its long conductors, inspection along conductors is preferred.

\section{Formation OF THE PICK-UP SignaL}

According to the Fig. 2, the output signal is composed of two components: the $U_{\text {offset }-1}$ component caused by an effect of the exciting current on the pick-up coil, and the $U_{\text {offset-2 }}$ component generated directly by the eddy currents. These two components have the same frequency but different phases. $U_{\text {offset-I }}$ and $U_{\text {offset-2 }}$ added together produce an offset signal $U_{\text {offset. }}$. 


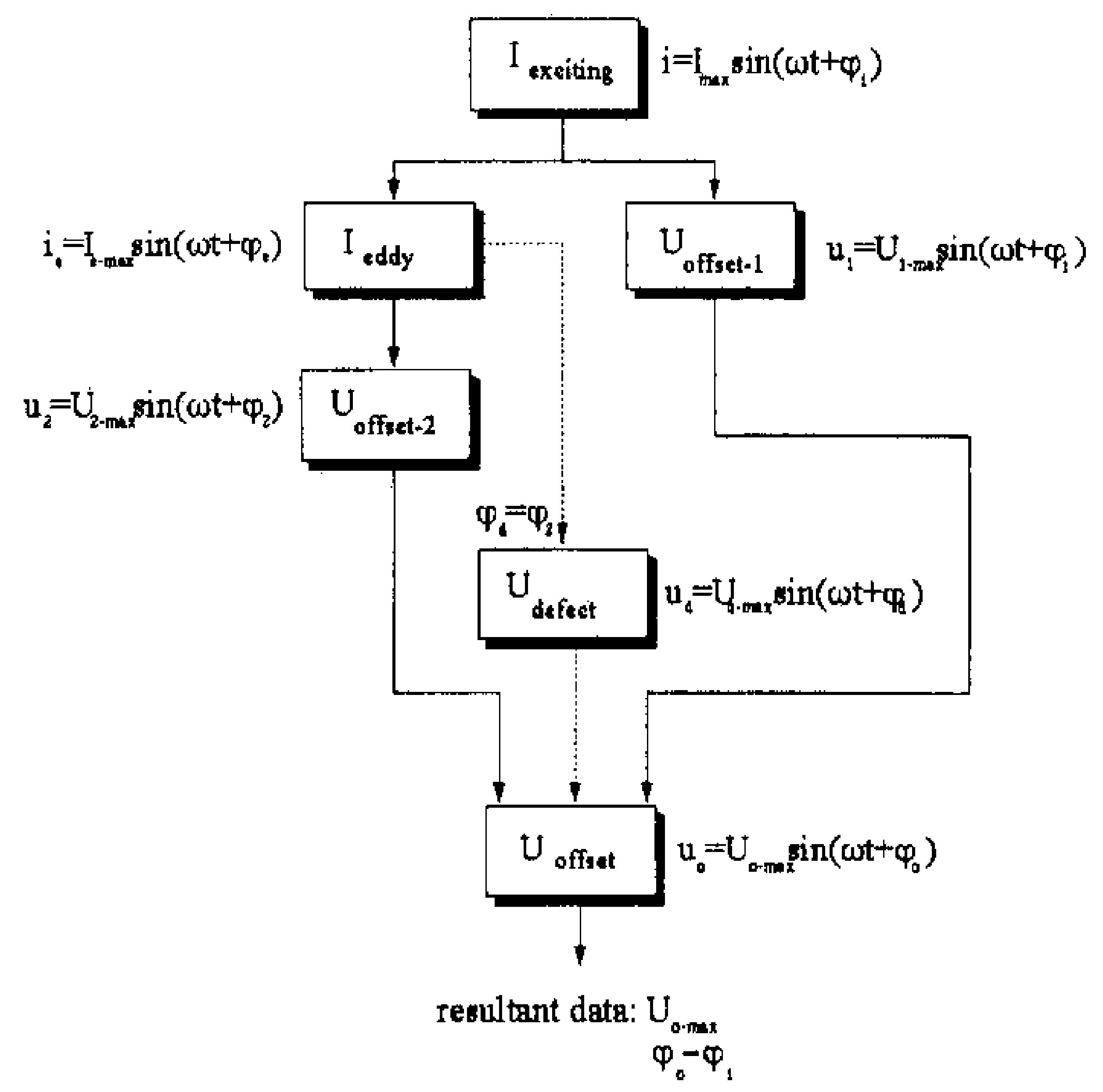

Fig. 2. Formation of the output signal during the inspection of PCB conductor by the ECT probe.

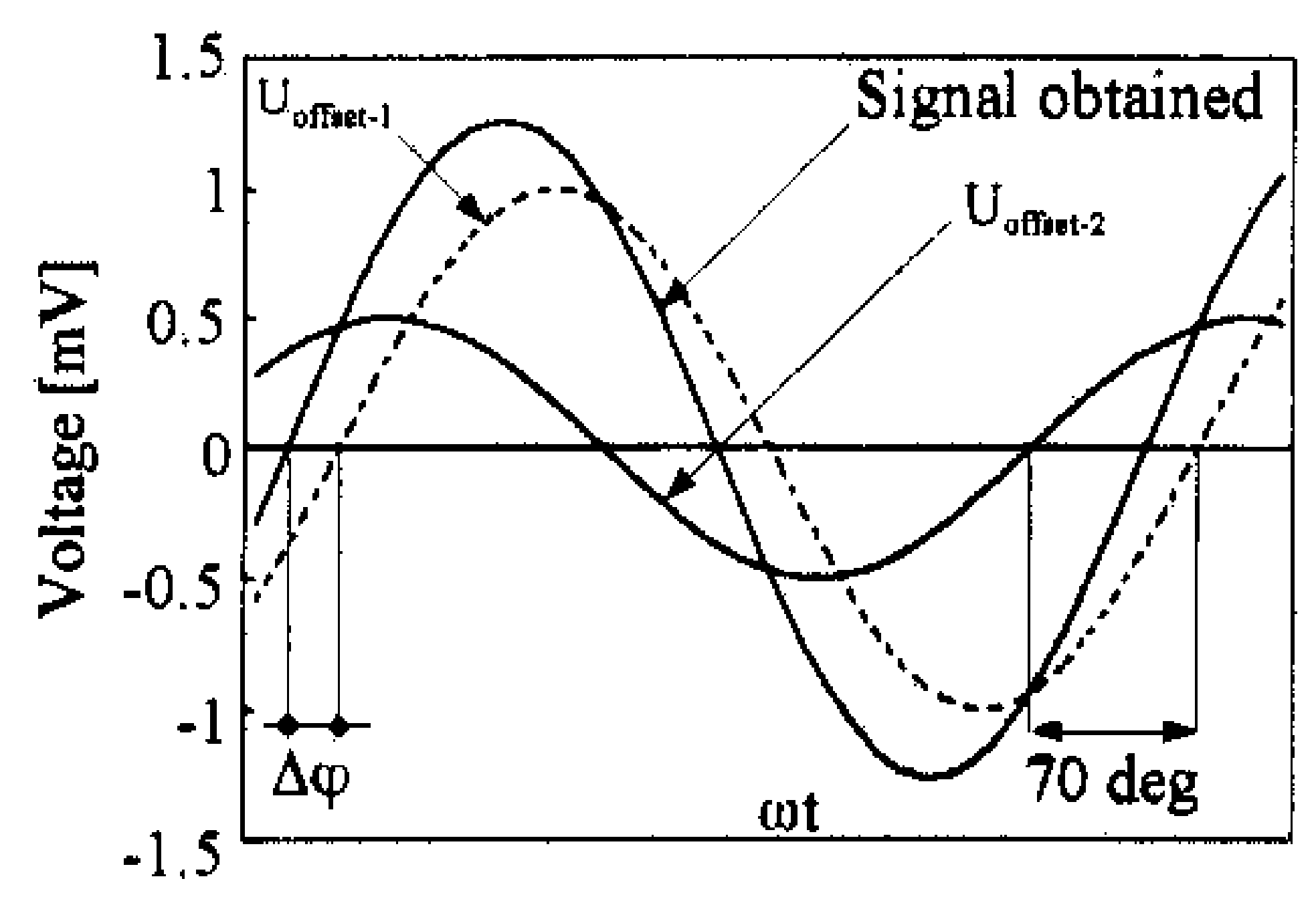

Fig. 3. Formation of the output signal from two sinusoidal components $U_{\text {offat }-1}$ and $U_{\text {offaet }-2}$.

During the inspection of a homogeneous material, the resultant data obtained from the lock-in amplifier $\left(U_{o-\max }\right.$ and $\left.\varphi_{o}-\varphi_{1}\right)$ remain constant. In case of the inspection of a Printed Circuit Board conductor with a defect, the amplitude $U_{2-\max }$ of the component $U_{\text {offset-2 }}$ increases by the amplitude $U_{d-\max }$ of a signal caused by the defect- $U_{\text {defect }}$. Fig. 3 shows the formation of the output signal obtained as a sum of two components $U_{\text {offset-1 }}$ and $U_{\text {offset-2 }}$. The components have sinusoidal character and, according to experimental results, there is $70^{\circ}$ difference between their phases.

\section{SimUlation of Amplitude ANd Phase Characteristics}

The amplitude and the phase of the output signal are effected by a proportion $P$ :

$$
P=\frac{U_{1-\max }}{U_{2-\max }+U_{d-\max }}
$$
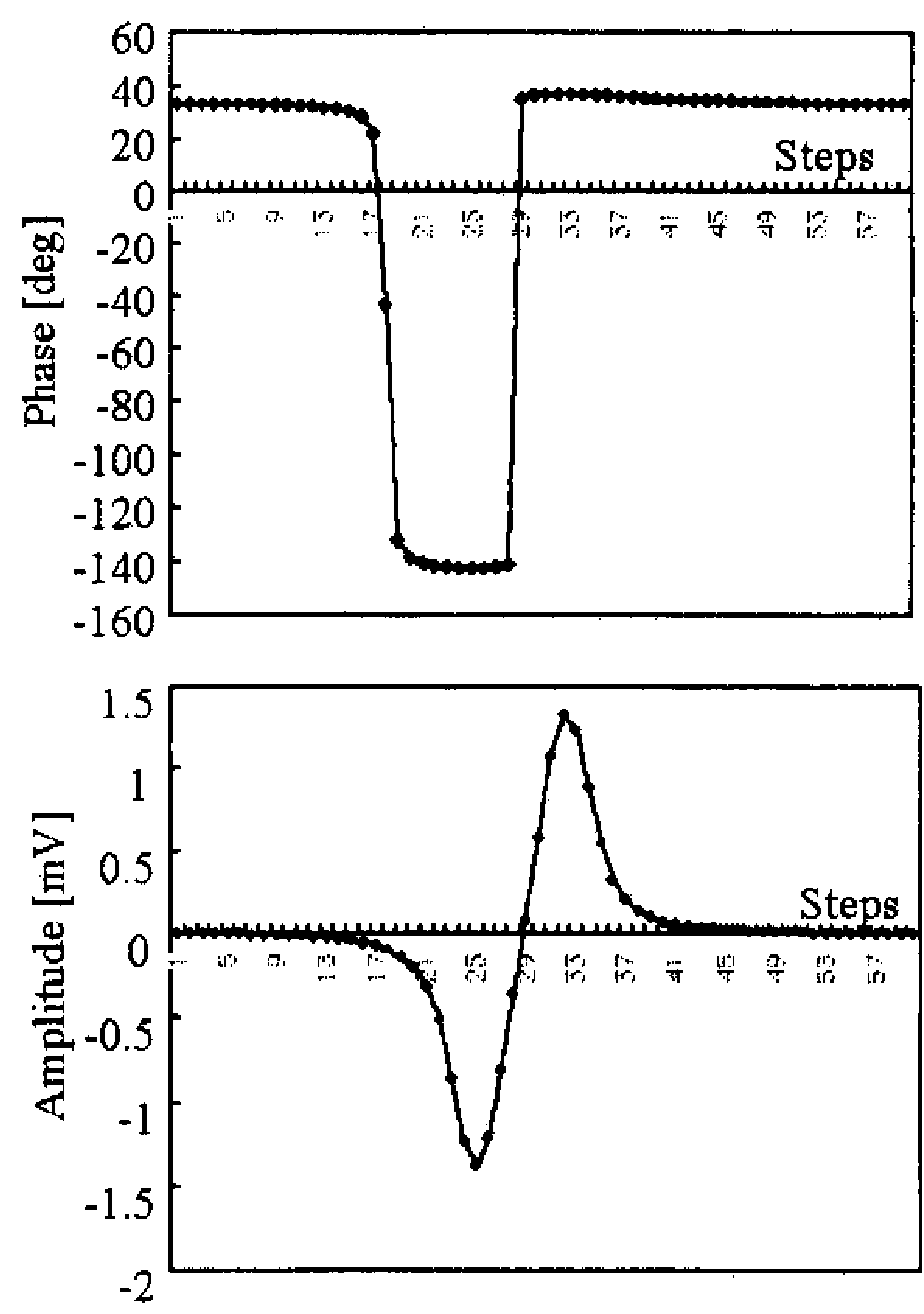

Fig. 4. Calculated phase and amplitude in case of: $P=0.2$.

where:

$U_{1-\max }$ is the amplitude of the component caused by the exciting current,

$U_{2-\max }$ is the amplitude of the component caused by the eddy currents,

$U_{d-\max }$ is the amplitude of the component due to the defect. The amplitude $U_{d-\text { max }}$ obtained during the inspection of a narrow $(0.2 \mathrm{~mm})$ conductor is much smaller than the amplitude $U_{d-\max }$ from the inspection of a wide conductor $(0.8 \mathrm{~mm})$ or a soldering point. Therefore the proportion $P$ strongly depends on the width of an inspected conductor. Characteristic "rectangular" changes of the phase, shown in Fig. 4, exist only during the inspection of wide conductors or soldering points, where $P$ is close to 0.2 .

The "rectangular" shape on the phase characteristic does not appear during the inspection of narrow conductors $(P \approx 5)$, where the amplitude $U_{\text {defect }}$ is too small to switch the phase of the total signal and only generates a "wave" form as shown in Fig. 5. The rectangular shape on the phase characteristic reflects a switching process between two values of the phase. A phenomenon of the switched phase may be used for an extraction of signals coming from soldering points from all signals obtained during the inspection. The signals representing soldering points are undesirable. Their elimination from a final image is a very important factor in the data analysis process. Fig. 6 shows a PCB model consisting of a soldering point and a conductor $0.2 \mathrm{~mm}$ wide. The component $U_{\text {defect }}$ is generated by the $\tan$ gential component of the magnetic flux density $B_{z}$. In the area of the defect, where the value of $B_{z}$ is relatively small due to the width of the conductor, the phase characteristic possesses a wave form, as shown in Fig. 7. The area of the soldering point, 

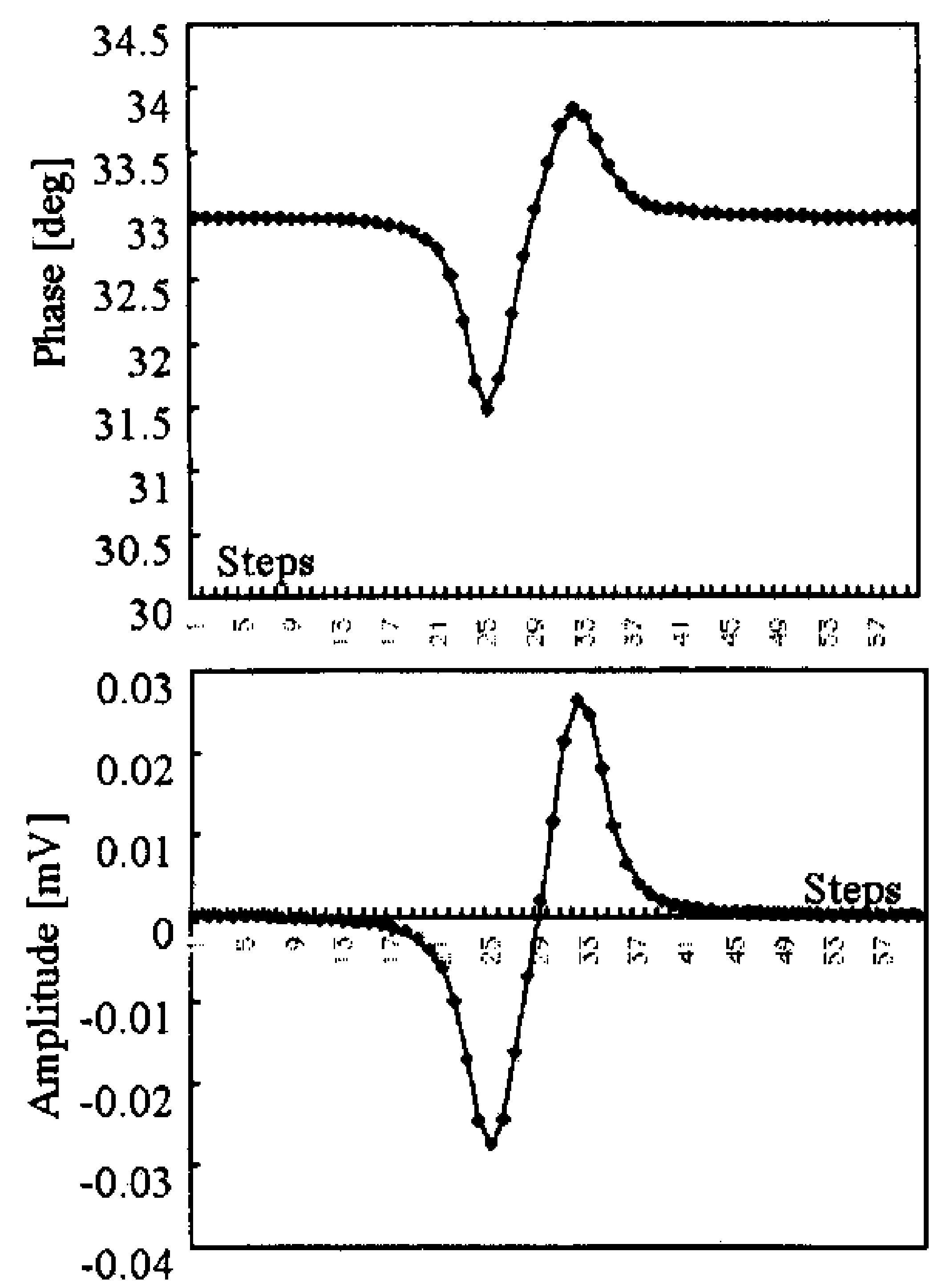

Fig. $\dot{5}$. Calculated phase and amplitude in case of: $P=5$.

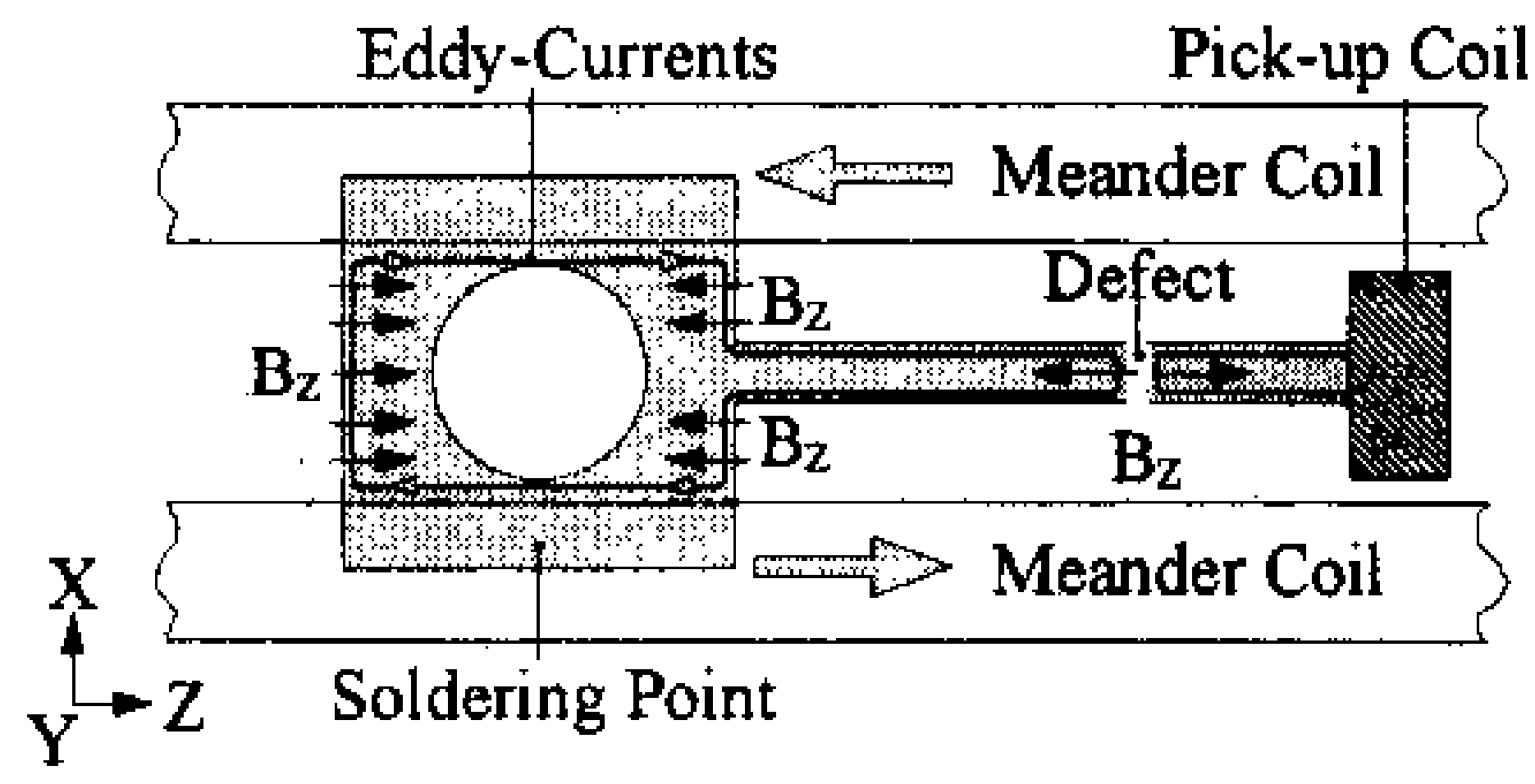

Fig. 6. PCB model-a soldering point and a narrow conductor.

where value of $B_{z}$ is much higher, can be seen as the rectangular shape on the phase characteristic.

\section{CONCLUSIONS}

A study of the output signal structure provides an understanding of the nature and factors causing changes in amplitude and phase characteristics. During the inspection of soldering

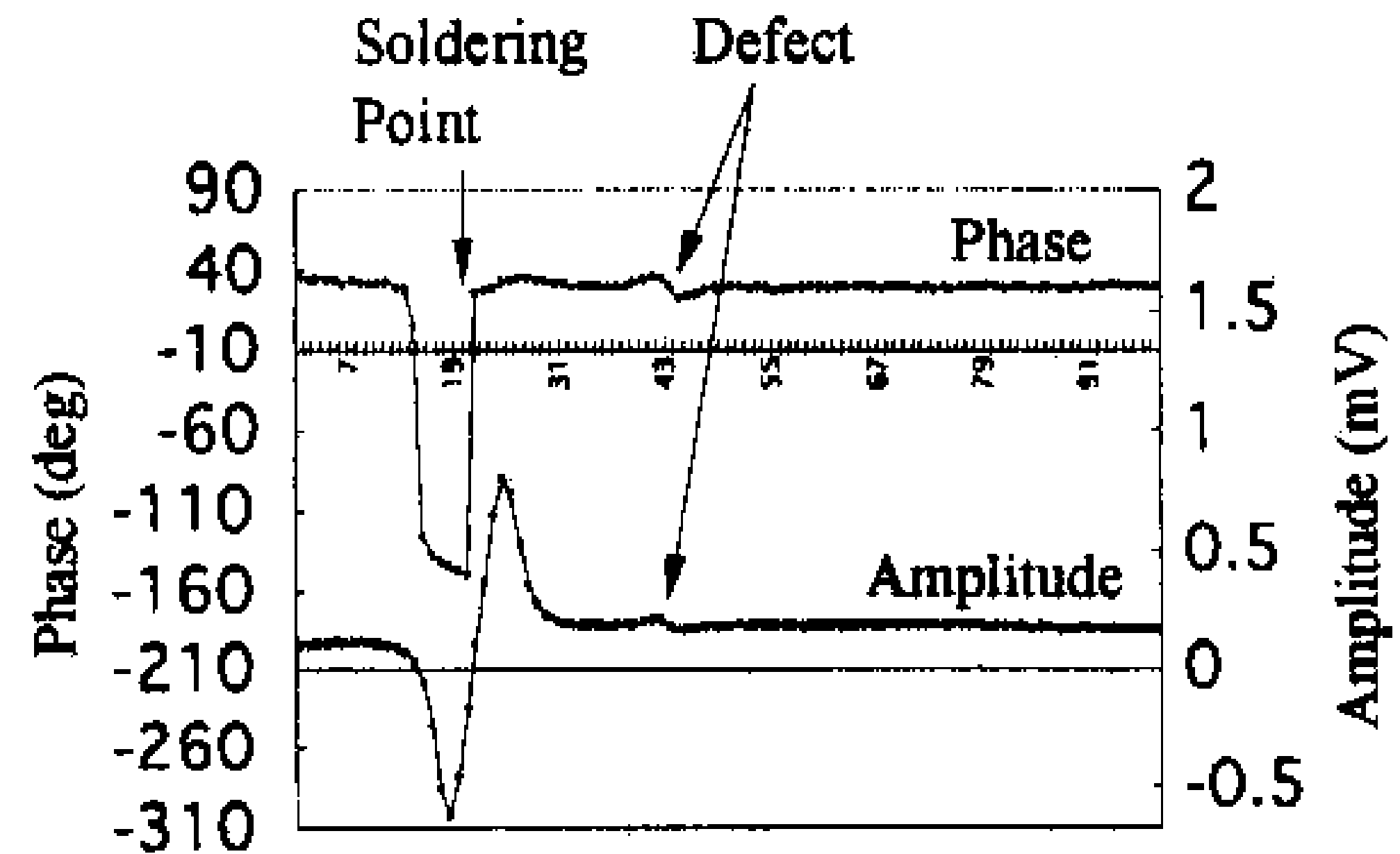

Fig. 7. Phase and amplitude characteristics obtained from the PCB model showed in Fig. 5-experimental results.

points, located on PCB, characteristic "rectangular" shapes on the phase characteristics were obtained. These "rectangular" shapes have been explained and confirmed by the numerical simulation. They exist not only during the inspection of soldering points. These changes can be also observed during the inspection of a metal surface with rivet holes. However for this inspection the size of the ECT probe has to be adjusted to keep proper proportion of the components.

The "rectangular" shapes on the phase characteristic inspire a new approach to the signal analysis, which is the classification of signals due to their shape.

\section{REFERENCES}

[1] D. Kacprzak, T. Miyagoshi, S. Yamada, and M. Iwahara, "Inspection of printed circuit board by ECT probe with solenoid pickup coil," Journal of J. Magn. Soc. Japan, vol. 24, no, 4-2, pp. 839-842, 2000.

[2] D. Kacprzak, S. Yamada, and M. Iwahara, "Analysis of the structure of printed circuit boards by means of the eddy current technique," $I N$ SIGHT Non-Destructive Testing and Condition Monitoring (The Journal of The British Institute of Non-Destructive Testing), vol. 42, no. 5, May 2000.

[3] D. Kacprzak, T. Miyagoshi, T. Tanigushi, S. Yamada, and M. Iwahara, "Comparison of two types of pick-up coil for meander excitation," in Non-Linear Electromagnetic Systems, P. Di Barba and A. Savini, Eds: IOS Press, 2000, pp. 229-232.

[4] T. Taniguchi, D. Kacprzak, S. Yamada, M. Iwahara, and T. Miyagoshi et al., "Defect detection of printed circuit board by using eddy current testing technique and image processing," in Electromagnetic Nondestructive Evaluation (IV), S. S. Udpa et al., Eds: IOS Press, 2000, pp. 111-119.

[5] T. Miyagoshi, D. Kacprzak, S. Yamada, and M. Iwahara, "Feasibility of inspecting defects in printed circuit boards by using eddy-current testing technique," Journal of Magnetic Society of Japan, vol. 23, no. 4-2, pp. 1613-1616, 1999.

[6] D. Kacprzak, T. Taniguchi, S. Yamada, M. Iwahara, and T. Miyagoshi, "Interpretation of printed circuit boards structure via amplitude and signal phase obtained from ECT testing," in 6th Int. Workshop on Electromagnetic Non-Destructive Evaluation, 2000. 